\title{
From the self-force problem to the radiation reaction formula
}

\author{
Yasushi Mino \\ Mail code 130-33 Caltech, Pasadena, CA 91125, USA \\ E-mail: mino@tapir.caltech.edu
}

Received 18 October 2004, in final form 7 January 2005

Published 18 July 2005

Online at stacks.iop.org/CQG/22/S717

\begin{abstract}
We review recent theoretical progress in the so-called self-force problem of a general relativistic two-body system. Although a two-body system in Newtonian gravity is a very simple problem, some fundamental issues are involved in relativistic gravity. Besides, because of recent projects for gravitational wave detection, it has become possible to see those phenomena directly via gravitational waves, and the self-force problem becomes one of urgent and highly-motivated problems in general relativity. Roughly speaking, there are two approaches to investigate this problem; the so-called postNewtonian approximation, and a black-hole perturbation. In this paper, we review theoretical progress in the self-force problem using a black-hole perturbation. Although the self-force problem seems to be just a problem to calculate a self-force, we discuss that the real problem is to define a gaugeinvariant concept of a motion in a gauge-dependent metric perturbation.
\end{abstract}

PACS numbers: $04.20 . \mathrm{Cv}, 04.25 . \mathrm{Nx}$

\section{Introduction}

For a single isolated body, the self-induced gravitational field does not accelerate the body because of momentum conservation. However, in a multi-body system in general relativity, there could be the case that the self-induced gravitational field could accelerate the body. Although one may naively say we have the self-force acting on the body, the concept of the body's motion is itself ambiguous in general relativity because one can freely choose a reference coordinate system to describe it. Thus, even if we could derive an explicit form of the self-force, there still remains a fundamental question; what we learn from the self-force about the body's motion. Here we focus on a binary system as a simple example to consider this fundamental question.

The dynamics of a binary system is also motivated by rapidly-progressing projects of gravitational wave detection. The so-called post-Newtonian approximation has a fairly long 
history in calculating the evolution of a equal-mass binary system. Although we now have a huge number of interesting results, the post-Newtonian approximation was usually done only with a few choices of the coordinate system.

Because the choice of coordinate systems is a key issue in understanding this fundamental question, it would be more interesting to consider another approach in which we may have more degrees of freedom to choose coordinate systems. Here we consider the self-force problem by a black-hole perturbation. We consider the relativistic two-body problem as a background black hole and a point particle orbiting around the black hole. We assume that the background black hole is described by a Kerr geometry, and consider a metric perturbation induced by a particle moving along a general bound orbit. The reference coordinate system for the particle's motion is dominantly given by the coordinate system of the background Kerr geometry, but, since the background geometry is perturbed by the self-gravity of the particle, we have a small freedom to choose the coordinate system. This is what we know as a gauge freedom of the metric perturbation.

This two-body system is considered to be a good approximation of a gravitationalwave source we may see with the LISA project. Because of the long base line, LISA will see low frequency gravitational waves (around $10 \mathrm{mHz}$ to $0.1 \mathrm{~Hz}$ ). Gravitational waves in this frequency range may be obtained from an orbit around a supermassive black hole (around $10^{6}-10^{8} M_{\odot}$ ), which we may astrophysically expect to exist at the centre of a galaxy. Our recent understanding of the dynamical structure of a galactic core suggests that supermassive black holes in the observable range of LISA would capture stellar mass compact objects in their gravitational potentials. Those gravitationally captured objects are inspiralling around the black holes, emitting gravitational waves detectable by LISA.

\section{Overview}

The study of orbital motion of a particle in a black-hole geometry started from the discovery of the so-called MiSaTaQuWa self-force [1], which we shall review in section 3. At this moment, the question was how we deal with a point particle and we obtained a general covariant expression of the self-force in a given vacuum background. Since the MiSaTaQuWa self-force is just a formal result, our next question was to evaluate the self-force explicitly in a Kerr geometry, using a known technique to calculate a metric perturbation. We review some basic ideas of some promising calculation methods in section 4 .

There are two approaches. One is to calculate the self-force directly by explicitly implementing the point splitting regularization of the MiSaTaQuWa self-force, which we call the self-force calculation. The other is to calculate the radiation reaction to the constants of motion based on our understanding of the self-force, and we call this the radiation reaction formula. By the self-force calculation, one may have the exact self-force in the framework of a linear perturbation, however, we still have a technical problem when we consider the self-force calculation in a Kerr black hole. Besides, as we shall discuss in section 5, there is a theoretical issue whether the self-force derived by this method correctly describes the radiation reaction effect. On the other hand, the radiation reaction formula considers radiation reaction to the orbit. Different from the self-force calculation, we only calculate the radiative part of the self-force in a class of gauge conditions where we are guaranteed to include the radiation reaction effect on the orbit. It has a technical advantage that the calculation method for this formula is already established. Furthermore, our recent result shows that the radiation reaction formula may have the most optimal prediction of the orbit. 
It is widely believed that the self-force describes the orbital evolution with the effect of gravitational radiation reaction. Section 5 discusses that this is not true in general. We point out that the usual scheme of a metric perturbation describes binary evolution only for a finite time interval. We show that the self-force could vanish by a special gauge transformation for this whole time interval, irrespective of the gravitational radiation we see from the asymptotic gravitational wave flux. Thus, strictly speaking, the self-force has nothing to do with the gravitational radiation reaction in the usual metric perturbation without some restriction of gauge conditions. We consider this problem happens not because of our definition of the self-force but because the usual scheme of a metric perturbation cannot describe the binary evolution long enough. In order to solve this, we propose an adiabatic extension of a metric perturbation, which we review in section 6 . The adiabatic extension is possible under a class of gauge conditions, where the self-force is guaranteed to include the radiation reaction effect. We also discuss the validity of the adiabatic approximation of the linear metric perturbation and the self-force defined by this. We find that the self-force in this class of gauge conditions might not predict the binary evolution sufficiently long for the LISA project in general. In order to extend the validity, we introduce a special gauge condition where the radiation reaction formula gives an exact self-force. In this gauge condition, we find that the self-force may predict the binary evolution long enough for the LISA project.

\section{Self-force}

In this section, we review a derivation of the MiSaTaQuWa self-force. In this paper, we focus on the physical motivation of our calculation strategy and we omit technical details of the calculation. We suggest that readers interested in those details refer to our original paper, or the review papers by us or Poisson [1].

\subsection{A historical motivation}

In a black-hole perturbation, a point particle is not well-defined because the self-gravity of the point particle diverges and the metric perturbation becomes invalid around the particle. Nevertheless, we had been using the point particle without any rigorous supporting argument because of the technical simplicity and because the point particle might be the only possible description of a star whose internal structure is supposed to be unimportant, and the metric perturbation induced by a point particle as being investigated for a long time [2] before the MiSaTaQuWa self-force was derived [1].

At this time, we used the so-called Press-Teukolsky formalism [3] which gives us a finite energy and angular momentum flux carried away by gravitational waves. Even if the point particle induces a divergent gravitational perturbation, one can define a finite energy and angular momentum on a $t$-constant hypersurface in the background Kerr geometry and we consider that the loss of the energy and angular momentum by the gravitational wave emission describes the radiation reaction to the particle's motion. This method is now referred to as the balance formula.

The balance formula was considered to be successful to describe radiation reaction to a particle moving along a quasi-circular orbit or an equatorial orbit. In this case, we consider an adiabatic approximation of the orbit where the orbit is approximated by a geodesic at each instant. Then, instead of the evolution of the orbital coordinates, we consider the evolution of 'three constants of motion' (the orbital energy $E$, the $z$ component of the angular momentum $L$ and Carter constant $C$ ) of geodesics around a Kerr geometry. The balance formula tells us the radiation reaction to only two of these constants, $E$ and $L$, and the problem was to derive 
the radiation reaction to the last, $C$. When the orbit is either quasi-circular or equatorial, one can prove that it will remain quasi-circular or equatorial under radiation reaction [4], as a result, we have an algebraic relation with $E, L$ and $C$ to derive radiation reaction to $C$ by the balance formula.

The difficulty in computing the radiation reaction to $C$ was that the Carter constant is not associated with the Killing vector. The Press-Teukolsky formalism was derived because $E$ and $L$ are the conserved currents defined on a $t$-constant hypersurface in association with the symmetry of the background spacetime. On the other hand, the Carter constant is defined only along a geodesic and has nothing to do with the symmetry. Besides, it came to be known that, in order to integrate the geodesic equation, one needs three phase constants in addition to the constants of motion, and we did not know how those phase constants evolve by gravitational radiation reaction.

At this moment, the simplest solution of this problem seemed to be an explicit calculation of the self-force. We considered that the self-force must have contained all the information of the orbit with the effect of radiation reaction, and that, after computing the orbit using the self-force, one can calculate a gravitational waveform by a linear metric perturbation formalism. As we shall argue in section 5, this statement is proven to be incorrect because the usual perturbation formalism cannot describe the radiation reaction to the orbit; nevertheless, this idea had been spread widely in the gravitational wave community.

\subsection{Problem of divergence}

A point particle is defined by the Dirac delta function. It has no volume, but a finite mass. This makes a divergent metric perturbation at the location of the particle. Because of the divergence, we have two problems;

(1) Can we use the point particle to induce a linear metric perturbation, which invalidates the linear perturbation scheme?

(2) Can we extract finite physical information on the self-force from the divergent field?

A common idea to understand a divergent field in quantum field theory is renormalization. In electromagnetism, a point charge induces a divergent vector potential at the location of the particle. When the charge moves in a curved spacetime, this self-induced vector potential will act on the charge's motion. Because quantum electrodynamics is renormalizable, a formal derivation of this electromagnetic self-force in a curved spacetime was successfully made by mass renormalization [5]. Here one evaluates a surface integration of the stress-energy tensor of the charge and the electromagnetic field over a world tube surrounding the orbit. The sum of the surface integral must vanish because of Gauss's law, and we obtain the self-force equation including the effect of electromagnetic radiation reaction.

We considered an analogous calculation for a gravitational self-force by mass renormalization [1]. We do not have the stress-energy tensor of the particle and the full gravitational field, however, we could find a conserved current of the particle and the metric perturbation in the background geometry. We consider expanding the Einstein tensor by the metric perturbation $h_{\mu \nu}$ in the background $g_{\mu \nu}$ as

$$
G_{\mu \nu}[g+h]=G_{\mu \nu}[g]+G_{\mu \nu}^{(1)}[h]+G_{\mu \nu}^{(2+)}[h],
$$

where $G_{\mu \nu}^{(1)}$ and $G_{\mu \nu}^{(2+)}$ represent the linear terms and all the nonlinear terms of the expansion with respect to $h_{\mu \nu}$. Because we assume the background is a vacuum solution, we have $G_{\mu \nu}[g]=0$. It is well known that the divergence of $G_{\mu \nu}^{(1)}[h]$ with respect to the background geometry vanishes algebraically. Thus, we have the conserved current in the background 
geometry as

$$
\mathcal{T}_{\mu \nu}=-G_{\mu \nu}^{(2+)}[h]+T_{\mu \nu}
$$

where $T_{\mu \nu}$ is the stress-energy tensor of the particle. Because $G_{\mu \nu}^{(2+)}[h]$ is quadratic in $h_{\mu \nu}$ to the leading order of the metric perturbation, we may consider this an 'effective stress-energy tensor' of the particle and the metric perturbation defined in the background geometry. By doing a surface integration over a world tube, we obtained a gravitational self-force equation by the mass renormalization.

Although we could have derived the MiSaTaQuWa self-force by mass renormalization, the derivation did not answer the above fundamental questions. A crucial issue in this derivation is that the perturbative quantum gravity is known to be nonrenormalizable; thus, there is no theoretical support for using mass renormalization in deriving the gravitational self-force. Furthermore, a physical interpretation of the motion is not clear yet. If we suppose that the 'point particle' represents a black hole, for example, the centre of the particle is inside the event horizon, and one may ask what it means to consider a motion hidden inside the horizon.

\subsection{Matched asymptotic expansion}

In order to answer these fundamental questions on the use of the 'point particle', we consider another approach. Since the primary problem here is a theoretical justification to use the 'point particle' rather than just a calculation of the self-force, we consider constructing a metric itself by using a matched asymptotic expansion technique. By the matched asymptotic expansion, we consider locating a small black hole of length scale $m$ in a background spacetime of curvature scale $L$. Assuming that $m \ll L$, one could construct the metric perturbatively by a small parameter $m / L \ll 1$. This approach was originally taken by the post-Newtonian calculation [6], where we have an explicit expression for a metric easy to control. In this problem, we consider locating the small black hole in a general vacuum background spacetime and, in order to have an explicit expression of the metric, we use the so-called bi-tensor formalism introduced in [5].

With a radial distance $r(>0)$ from the centre of the small black hole, the gravitational field is determined dominantly by that of the small black hole at $r<L$, and by the background spacetime at $r>m$. Hence, the metric at $r<L$ is constructed by the small black hole with a black-hole perturbation (which we call the near-zone expansion) as

$$
g_{\mu \nu}^{\text {near }}=g_{\mu \nu}^{B H}+L^{-1} g_{\mu \nu}^{(1 /)}+L^{-2} g_{\mu \nu}^{(2 /)}+\cdots,
$$

where $g_{\mu \nu}^{B H}$ is the metric of the small black hole. The black-hole perturbation $g_{\mu \nu}^{(n /)}$ is defined with an appropriate boundary condition on the black-hole horizon. As we cannot specify a boundary condition for $g_{\mu \nu}^{(n /)}$ at $r \geqslant L$, the metric perturbation cannot be determined uniquely until we complete the matching. The metric at $r>m$ is constructed by the background spacetime with its perturbation (we call the far-zone expansion) as

$$
g_{\mu \nu}^{\mathrm{far}}=g_{\mu \nu}^{b g}+m g_{\mu \nu}^{[/ 1]}+m^{2} g_{\mu \nu}^{[/ 2]}+\cdots,
$$

where $g_{\mu \nu}^{b g}$ is the metric of the background spacetime. The perturbation of the background $g_{\mu \nu}^{[/ n]}$ is derived with an appropriate asymptotic boundary condition and is not determined uniquely because of the lack of a boundary condition at $r \leqslant m$. Instead of the boundary condition at $r \leqslant m$, one can put auxiliary matter at $r \leqslant m$ to induce a vacuum perturbation at $r>m$, and we use a point source with an arbitrary internal structure derived in [7].

The matched asymptotic expansion can be done at the radius where both near-zone and far-zone expansions are valid, which we call the overlapping zone. Suppose we take the radius 
of the overlapping zone as $r \sim \sqrt{m L}$, and one can re-expand (3.3) and (3.4) as

$$
\begin{aligned}
& g_{\mu \nu}^{\text {near }}=\left(g_{\mu \nu}^{(0 / 0)}+m g_{\mu \nu}^{(0 / 1)}+\cdots\right)+L^{-1}\left(g_{\mu \nu}^{(1 / 0)}+m g_{\mu \nu}^{(1 / 1)}+\cdots\right)+\cdots, \\
& g_{\mu \nu}^{\mathrm{far}}=\left(g_{\mu \nu}^{[0 / 0]}+L^{-1} g_{\mu \nu}^{[1 / 0]}+\cdots\right)+m\left(g_{\mu \nu}^{[0 / 1]}+L^{-1} g_{\mu \nu}^{[1 / 1]}+\cdots\right)+\cdots
\end{aligned}
$$

From a dimensional analysis, we may see $g_{\mu \nu}^{(n / m)} \sim g_{\mu \nu}^{[n / m]} \sim r^{n-m}$, thus, at the overlapping zone, one can estimate the expansions as

$$
L^{-n} m^{m} g^{(n / m)} \sim L^{-n} m^{m} g^{[n / m]} \sim(m / L)^{(n+m) / 2} .
$$

Under the matched asymptotic expansion, we assume $m / L \ll 1$ and we equate $g_{\mu \nu}^{(n / m)}=g_{\mu \nu}^{[n / m]}$ one by one from small to large $n+m$ which will perturbatively determine the boundary condition of (3.3) at $r \geqslant L$ and the internal structure of the auxiliary matter to induce the perturbation of (3.4).

Here, it is important to note coordinate conditions. Since the derivations of (3.3) and (3.4) are totally independent, they may use different coordinate systems, and we have to introduce a coordinate transformation so that we can correctly evaluate $g_{\mu \nu}^{(n / m)}=g_{\mu \nu}^{[n / m]}$. It is also important to fix a gauge condition for the metric of the small black hole in order to fix the location of the particle in the near-zone expansion.

We suppose that the background metric and its coordinate system are given by $\left(g_{\alpha \beta}^{b g},\left\{x^{\alpha}\right\}\right)$ and that the orbit in the background metric is described by $z^{\alpha}(\tau)$ with a parameter $\tau$. We also suppose that the black-hole metric and its coordinate system are given by $\left(g_{i j}^{B H},\left\{X^{i}, i=0,1,2,3\right\}\right)$ and we take a Schwarzschild black-hole metric in the harmonic coordinates where $X^{0}$ is the temporal coordinate and $X^{1}, X^{2}, X^{3}$ are spatial. In order to fix the location of the small black hole in the near-zone expansion, we take the gauge condition such that the $l=1$ modes of the perturbation $L^{-n} g_{i j}^{(n /)}$ in the tensor-spherical decomposition vanish. Then, we may say the particle is located at $X^{1}=X^{2}=X^{3}=0$ in the near-zone expansion.

For the matched asymptotic expansion of the metrices in the far-zone expansion and the near-zone expansion, we define the coordinate transformation by using a tetrad defined along the orbit and derivatives of the bi-scalar of the half-the-squared geodesic distance. The coordinate transformation is determined perturbatively by the matched asymptotic expansion of the metrices.

\subsection{MiSaTaQuWa self-force}

We find that the coordinate transformation gives us the information on the particle orbit because it relates the location of the small black hole in the near-zone expansion (i.e. $X^{1}=X^{2}=X^{3}=0$ ) to the coordinates in the far-zone expansion. By matching $g_{\mu \nu}^{(0 / 0)}=g_{\mu \nu}^{[0 / 0]}$ and $g_{\mu \nu}^{(1 / 0)}=g_{\mu \nu}^{[1 / 0]}$, we find that the particle moves along a geodesic. By the matching $g_{\mu \nu}^{(2 / 0)}=g_{\mu \nu}^{[2 / 0]}, g_{\mu \nu}^{(0 / 1)}=g_{\mu \nu}^{[0 / 1]}, g_{\mu \nu}^{(1 / 1)}=g_{\mu \nu}^{[1 / 1]}$ and $g_{\mu \nu}^{(2 / 1)}=g_{\mu \nu}^{[2 / 1]}$, we derive the self-force.

The point particle is shown to be consistent for the derivation of the metric in the far-zone expansion as a result of the matched asymptotic expansion of the metric. Even though the point particle makes a divergent metric perturbation, it is just an auxiliary source to induce a finite metric perturbation at $r>m$ in the far-zone expansion. This justifies the use of a point particle in calculating an energy and angular momentum flux via an asymptotic metric perturbation since it uses the metric perturbation only in the far-zone expansion.

We also consider that the derivation of the self-force by the matched asymptotic expansion suggests a physical interpretation of the particle motion. In the end, the motion of the particle 
is considered by the coordinate system in the far-zone expansion which is the background metric plus a small perturbation induced by the small black hole. For an observer far from the particle, the background metric dominantly determines a reference frame to measure the motion of the particle with, whatever non-perturbative things happen around the small black hole, thus, the self-force derived by the matched asymptotic expansion is expected to be physically meaningful for such an observer. Because the small black hole is small compared to the curvature length of the background metric, one can assign the location of the particle in the coordinate system of the regular background metric, and consider the motion of the particle without divergence. Here we have a small ambiguity in this assignment of the particle location because of a finite size of the small black hole, which can be interpreted as a gauge ambiguity of the self-force. The location of the black hole in the near-zone metric is determined by the gauge condition of the metric perturbation $L^{-n} g_{\alpha \beta}^{(n /)}$. In the present derivation of the self-force, the gauge conditions of $L^{-1} g^{(1 / 1)}$ and $L^{-2} g_{i j}^{(2 / 1)}$ are important. By the matched asymptotic expansion, they fix the gauge condition of the linear metric perturbation $m g_{\alpha \beta}^{[/ 1]}$ of the far-zone expansion.

\section{Regularization calculation}

Since the MiSaTaQuWa self-force is just a formal expression of a self-force in an arbitrary background, the next problem was to explicitly calculate a self-force in a specific background, especially, a Kerr black hole for an application to the LISA project. The formal result of the self-force [1] can be written as

$$
\begin{aligned}
& F^{\alpha}(\tau)=\lim _{x \rightarrow z(\tau)} F^{\alpha}\left[h^{\mathrm{reg}}(x)\right], \\
& F^{\alpha}[k(x)]=\delta \Gamma_{\beta \gamma}^{\alpha}[k(x)] v^{\beta} v^{\gamma}+v^{\alpha} \delta \Gamma_{\beta \gamma \delta}[k(x)] v^{\beta} v^{\gamma} v^{\delta}, \\
& h_{\alpha \beta}^{\mathrm{reg}}(x)=h_{\alpha \beta}(x)-h_{\alpha \beta}^{\text {sing }}(x), \quad\left(x^{\alpha} \neq z^{\alpha}(\tau)\right),
\end{aligned}
$$

where $x^{\alpha}$ is a field point, $z^{\alpha}(\tau)$ is an orbit and $v^{\alpha}$ is the 4 -velocity. $\delta \Gamma_{\beta \gamma}^{\alpha}$ is a linear tensor differential operator acting on a metric perturbation to calculate a perturbed Christoffel symbol. $h_{\alpha \beta}(x)$ is a linear metric perturbation induced by a point particle, and $h_{\alpha \beta}^{\text {sing }}(x)$ is a singular part derived by the Hadamard expansion. $h_{\alpha \beta}^{\text {reg }}(x)$ is called a regularized metric perturbation. Both $h_{\alpha \beta}(x)$ and $h_{\alpha \beta}^{\text {sing }}(x)$ are divergent along the orbit, but $h_{\alpha \beta}^{\text {reg }}(x)$ is regular along the orbit since the divergences of $h_{\alpha \beta}(x)$ and $h_{\alpha \beta}^{\text {sing }}(x)$ cancel each other. Thus, the calculation of the self-force can be done by first evaluating it at a field point $x^{\alpha}$, then taking the limit $x^{\alpha} \rightarrow z^{\alpha}(\tau)$. By doing this point splitting regularization, we have a finite result.

There are two promising approaches. One is to calculate the self-force directly by doing a harmonic mode decomposition, which we call the self-force calculation. The other is to calculate the radiation reaction to the constants of motion based on our theoretical understanding of the self-force, which we call the radiation reaction formula.

\subsection{Self-force calculation}

Here we briefly review a method to compute a self-force directly. The basic idea here is that, since it is difficult to compute $F^{\alpha}\left[h^{\text {reg }}(x)\right]$ of (4.1) directly, we consider calculating $F^{\alpha}[h(x)]$ and $F^{\alpha}\left[h^{\operatorname{sing}}(x)\right]$, then the self-force can be derived by

$$
F^{\alpha}(\tau)=\lim _{x \rightarrow z(\tau)}\left(F^{\alpha}[h(x)]-F^{\alpha}\left[h^{\text {sing }}(x)\right]\right) .
$$


We call $F^{\alpha}[h(x)]$ and $F^{\alpha}\left[h^{\text {sing }}(x)\right]$ a bare self-force field and a singular self-force field, which are defined at a field point $x^{\alpha}$ not on the orbit $z^{\alpha}(\tau)$. It is important to note that the subtraction of (4.4) must be done before taking the coincidence limit $x^{\alpha} \rightarrow z^{\alpha}(\tau)$ since $F^{\alpha}[h(x)]$ and $F^{\alpha}\left[h^{\text {sing }}(x)\right]$ are divergent along the orbit.

This type of calculation is called regularization. Various techniques for regularization calculations are well-known in quantum field theory, but we cannot use those techniques for two reasons;

(1) In a usual regularization of quantum field theory, we consider a theory of global Lorentz invariance and we can expand the divergent field by the Fourier mode, respecting the Lorentz symmetry. However, a black-hole spacetime does not have such a symmetry and we may only decompose $h_{\alpha \beta}(x)$ into a Fourier-harmonic series.

(2) $h_{\alpha \beta}^{\text {sing }}(x)$ is derived by the Hadamard expansion, thus we have its local divergent expression only around the orbit.

Besides, in the case of a Kerr black hole as a background, a method to calculate a linear metric perturbation was proposed just recently in [8], and it has not been coded yet. Another simple technique to calculate a metric perturbation was proposed in [9]; however, it is known to be valid only for a homogeneous metric perturbation and it cannot describe a metric perturbation around the orbit. It is also important to note that the original self-force was derived in the harmonic gauge condition; thus, we may have a residual divergence by a difference of gauge conditions unless we calculate $h_{\alpha \beta}(x)$ in the harmonic gauge condition.

The most successful method at present is the so-called mode decomposition regularization [10], which is actually based on two ideas. One is to use a local coordinate expansion originally proposed by us [11], and the other is to use the so-called regularization parameters originally proposed by Barack, Burko and Ori [12]. Here we briefly review a basic idea of this regularization calculation in the case that the background is a Schwarzschild black hole. There is also some progress on an extension to a Kerr black hole and a method to deal with the residual divergence by a gauge condition, but we do not discuss it here because of its technicality.

We use the Schwarzschild coordinates and denote a field point and an orbital point by $x^{\alpha}=\{t, r, \theta, \phi\}$ and $z^{\alpha}=\left\{t_{0}, r_{0}, \theta_{0}, \phi_{0}\right\}$. We suppose that a full metric perturbation is derived by a harmonic decomposition and we calculate a harmonic decomposition of the bare self-force field ${ }^{1}$.

After the harmonic decomposition, the expansion coefficients become finite functions of $(t, r)$, and the divergence of the bare self-force field appears only by taking the infinite sum over harmonics at the coincidence limit $x^{\alpha} \rightarrow z^{\alpha}$. We take the angular coincidence limit $(\theta, \phi) \rightarrow\left(\theta_{0}, \phi_{0}\right)$, and take the sum over the azimuthal modes. Then the mode decomposition of the bare self-force field can schematically be written as

$$
F^{\alpha}[h]=\sum_{l} F_{l}^{(\text {bare }) \alpha}(t, r) .
$$

We note that the harmonic decomposition is ill-defined on a 2-sphere of $(t, r)=\left(t_{0}, r_{0}\right)$ because the bare self-force field diverges; as a result, the mode decomposition is not continuously defined across this 2-sphere as $\lim _{t \rightarrow t_{0}, r \rightarrow r_{0}+0} F_{l}^{\text {(bare) } \alpha} \neq \lim _{t \rightarrow t_{0}, r \rightarrow r_{0}-0} F_{l}^{\text {(bare) } \alpha}$.

As for the singular self-force field to be subtracted, we only have its local expression by the Hadamard expansion, and it is not defined on the entire 2-sphere $(\theta, \phi)$; hence, an exact

\footnotetext{
1 Since we are interested in calculating a self-force vector, there are some options in the harmonic decomposition. In order to avoid a complication of multiple components, we consider it easier to decompose each component of the self-force vector by the scalar harmonics. For this technical detail, see [10].
} 
harmonic decomposition cannot be defined. However, the regularization calculation (4.1) depends only on the local behaviour of the fields around the orbit, and one can freely add a finite term to the singular self-force field if it vanishes at the coincidence limit $x^{\alpha} \rightarrow z^{\alpha}$. We consider the local coordinate expansion of the singular self-force field. We suppose that $x^{\alpha}-z^{\alpha}$ is sufficiently small and expand the singular self-force field by the power of $x^{\alpha}-z^{\alpha}$ as

$$
\begin{aligned}
& F^{\alpha}\left[h^{\text {sing }}\right]=\sum_{n, n_{\alpha}} f_{\left(n, n_{\alpha}\right)}^{\alpha} \frac{1}{\epsilon^{n / 2}}\left(t-t_{0}\right)^{n_{t}}\left(r-r_{0}\right)^{n_{r}}\left(\theta-\theta_{0}\right)^{n_{\theta}}\left(\phi-\phi_{0}\right)^{n_{\phi}}, \\
& \epsilon=\frac{1}{2} g_{\alpha \beta}(z)\left(x^{\alpha}-z^{\alpha}\right)\left(x^{\beta}-z^{\beta}\right),
\end{aligned}
$$

where $g_{\alpha \beta}(z)$ is the background metric evaluated at the orbital location. For the regularization calculation, it is sufficient to decompose the non-vanishing terms of (4.6), i.e. only the terms of $n_{t}+n_{r}+n_{\theta}+n_{\phi}-n \leqslant 0$. Necessary formulae are developed in [10], and we have the mode decomposition of the singular self-force field schematically with vanishing ambiguous terms as

$$
F^{\alpha}\left[h^{\text {sing }}\right]=\sum_{l} F_{l}^{\left(\text {sing }^{\prime}\right) \alpha}(t, r) .
$$

(Because of vanishing ambiguous terms, the LHS and RHS are not exactly equal.) Again, the expansion coefficients are not continuous at the coincidence limit $(t, r) \rightarrow\left(t_{0}, r_{0}\right)$.

We subtract the singular self-force field from the bare self-force field. Because the singular behaviour of these fields cancel each other, the sum of the harmonics becomes finite even after taking the coincidence limit; thus, the self-force is derived by

$$
F^{\alpha}=\sum_{l}\left\{F_{l}^{(\text {bare }) \alpha}\left(t_{0}, r_{0}\right)-F_{l}^{\left(\text {sing }^{\prime}\right) \alpha}\left(t_{0}, r_{0}\right)\right\} .
$$

This shows that all we need in the end are the expansion coefficients at the coincidence limit $(t, r) \rightarrow\left(t_{0}, r_{0} \pm 0\right)$, where the limit must be taken consistently.

\subsection{Radiation reaction formula}

The primary question to motivate the radiation reaction formula is the relation between the self-force and the balance formula. As we argue for the self-force calculation in section 4.1, a complicated regularization calculation is necessary to derive the self-force, while the balance formula is simply formulated without regularization. We also have a question which part of the self-force is described in the balance formula. We find the relation of the self-force and the balance formula in [13], and review some basic arguments here. We also refer to [14] for a hint to answer these questions, where the energy and angular momentum loss of the balance formula are derived from the radiative part of the self-force by taking an infinite time average.

For preparation to discuss the radiation reaction formula, we first argue on some symmetry property of a Kerr geometry and geodesics. Using constants of motion $\mathcal{E}^{a}, a=\left\{E, L_{z}, C\right\}$, the geodesic equation in the Boyer-Lindquist coordinates becomes

$$
\begin{aligned}
& \left(\frac{\mathrm{d} r}{\mathrm{~d} \lambda}\right)^{2}=R\left(\mathcal{E}^{a} ; r\right), \quad\left(\frac{\mathrm{d} \theta}{\mathrm{d} \lambda}\right)^{2}=\Theta\left(\mathcal{E}^{a} ; \theta\right), \\
& \frac{\mathrm{d} t}{\mathrm{~d} \lambda}=T_{r}\left(\mathcal{E}^{a} ; r\right)+T_{\theta}\left(\mathcal{E}^{a} ; \theta\right), \quad \frac{\mathrm{d} \phi}{\mathrm{d} \lambda}=\Phi_{r}\left(\mathcal{E}^{a}, r\right)+\Phi_{\theta}\left(\mathcal{E}^{a} ; \theta\right) .
\end{aligned}
$$


As we are interested in the inspiral stage of the binary, we only consider the case that $r$-motion is bounded, then the $r$ - and $\theta$-motions become periodic [15] and we can expand them by discrete Fourier series as

$$
z^{b}=\sum_{n} Z^{b(n)} \exp \left[\mathrm{in} \chi_{b}\right], \quad \chi_{b}=2 \pi \tilde{\Omega}_{b}\left(\lambda+\lambda^{b}\right),
$$

where $b=\{r, \theta\}$ and $r=z^{r}, \theta=z^{\theta}$ and $Z^{b(n)}$ and $\tilde{\Omega}_{b}$ are functions of $\mathcal{E}^{a}$. Here we have two integral constants $\lambda^{b}$, but, since we can freely choose the zero point of $\lambda$, only $\lambda^{r}-\lambda^{\theta}$ can specify the geodesic. Because of these periodicities, one can freely add $1 / \tilde{\Omega}_{b}$ to $\lambda_{b}$. Suppose that the ratio of $\tilde{\Omega}_{r}$ to $\tilde{\Omega}_{\theta}$ is irrational, one can set $\lambda^{r}-\lambda^{\theta}$ as small as possible, then, by an appropriate choice of the zero point of $\lambda$, one can have $\lambda^{r}=\lambda^{\theta}=0$. $t$ - and $\phi$-motions are simply integrated as

$$
z^{c}=\kappa_{c}+\sum_{b, n} Z_{b}^{c(n)} \exp \left[\mathrm{in} \chi_{b}\right], \quad \kappa_{c}=\left\langle\dot{Z}^{c}\right\rangle \lambda+C^{c},
$$

where $c=\{t, \phi\}$ and $t=z^{t}, \phi=z^{\phi}$, and $\left\langle\dot{Z}^{c}\right\rangle$ and $Z_{b}^{c(n)}$ are functions of $\mathcal{E}^{a}$. Here we have two integral constants $C^{c}$ which specify the geodesic. Below, we call $\mathcal{E}^{a}$ the primary constants and $\lambda^{b}$ and $C^{c}$ the secondary constants of geodesics. The Kerr geometry has the $t$ and $\phi$-translation symmetries and, by these properties, one can set $C^{c}=0$.

We find an interesting symmetry of the Kerr geometry. The metric is invariant under

$$
t \rightarrow-t, \quad r \rightarrow r, \quad \theta \rightarrow \theta, \quad \phi \rightarrow-\phi .
$$

By this symmetry, the orbital parameter and the primary and secondary constants of a geodesic transform as

$$
\lambda \rightarrow-\lambda, \quad \mathcal{E}^{a} \rightarrow \mathcal{E}^{a}, \quad \lambda^{b} \rightarrow-\lambda^{b}, \quad C^{c} \rightarrow-C^{c} .
$$

Since the secondary constants can be set to zero, the geodesic is invariant under this symmetry transformation and we call this the geodesic preserving symmetry.

We next discuss the self-force. Because the self-force is gauge dependent, one has to specify a gauge condition for this discussion. Here we restrict to a class of gauge conditions such that the metric perturbation is derived as

$$
h_{\mu \nu}(x)=\int \mathrm{d} x^{\prime} G_{\mu \nu \mu^{\prime} \nu^{\prime}}\left(t-t^{\prime}, \phi-\phi^{\prime} ; r, r^{\prime}, \theta, \theta^{\prime}\right) T^{\mu^{\prime} v^{\prime}}\left(x^{\prime}\right),
$$

where the Green function has an appropriate fall-off condition at $\left|t-t^{\prime}\right|,\left|r-r^{\prime}\right| \rightarrow \infty$. We call this the physically reasonable class of gauge conditions because the Green function is invariant by the $t$ - and $\phi$-translation symmetries of the Kerr geometry, and because one can naturally read out spectral information on the asymptotic gravitational waves in this class of gauge conditions. Instead of the coordinate components of the self-force, we consider the evolution of the 'primary constants' by the self-force. The primary constants of a geodesic are defined by the Killing vectors $\eta_{\alpha}^{E / L}$ and tensor $\eta_{\alpha \beta}^{C}$ as $E=\eta_{\alpha}^{E} v^{\alpha}, L=\eta_{\alpha}^{L} v^{\alpha}, C=\eta_{\alpha \beta}^{C} v^{\alpha} v^{\beta} / 2$, and we consider the self-force acting on the 'primary constants' $\tilde{F}^{a}=\mathrm{d} \mathcal{E}^{a} / \mathrm{d} \lambda$ derived as

$$
\tilde{F}^{E}=\eta_{\alpha}^{E} \frac{\mathrm{D}}{\mathrm{d} \lambda} v^{\alpha}, \quad \tilde{F}^{L}=\eta_{\alpha}^{L} \frac{\mathrm{D}}{\mathrm{d} \lambda} v^{\alpha}, \quad \tilde{F}^{C}=\eta_{\alpha \beta}^{C} v^{\alpha} \frac{\mathrm{D}}{\mathrm{d} \lambda} v^{\beta} .
$$

We note that (4.10) and (4.11) rely only on the definitions of $\mathcal{E}^{a}$ whether they are constants or not; thus, (4.10), (4.11) and (4.17) describe the orbital evolution by the self-force. Because the self-force is induced by a geodesic to the leading order, it is a function of the primary and secondary constants and the orbital parameters. We find that the self-force does not depend on $C^{c}$ because of the $t$ - and $\phi$-translation symmetries; thus, we have $\tilde{F}^{a}=\tilde{F}^{a}\left(\mathcal{E}^{a}, \lambda^{b}, \lambda\right)$. Under 
the gauge condition defined by (4.16), we find the self-force could be expanded by discrete Fourier series as

$$
\tilde{F}^{a}=\sum_{n_{r}, n_{\theta}} \dot{\mathcal{E}}^{a\left(n_{r}, n_{\theta}\right)} \exp \left[\mathrm{i} n_{r} \chi_{r}+\mathrm{i} n_{\theta} \chi_{\theta}\right] .
$$

We consider applying the geodesic preserving symmetry transformation (4.14) to the selfforce. Because the transformation changes the direction of time, it also changes the boundary condition to derive the self-force. We derive the key identity,

$$
\tilde{F}^{\text {(ret)a }}\left(\mathcal{E}^{a}, \lambda^{b} ; \lambda\right)=-\tilde{F}^{(\text {adv }) a}\left(\mathcal{E}^{a},-\lambda^{b} ;-\lambda\right),
$$

where we denote the retarded boundary condition and the advanced boundary condition by (ret) and (adv) respectively to indicate the boundary condition for the self-force. Using the general form of the self-force (4.18), we have $\dot{\mathcal{E}}^{(\mathrm{ret}) a\left(n_{r}, n_{\theta}\right)}=-\dot{\mathcal{E}}^{(\mathrm{adv}) a\left(-n_{r},-n_{\theta}\right)}$. Suppose we calculate the self-force by the radiative (half-retarded-minus-half-advanced) Green function, we would have

$$
\frac{1}{2}\left(\dot{\mathcal{E}}^{(\mathrm{ret}) a\left(n_{r}, n_{\theta}\right)}+\dot{\mathcal{E}}^{(\mathrm{ret}) a\left(-n_{r},-n_{\theta}\right)}\right)=\dot{\mathcal{E}}^{(\mathrm{rad}) a\left(n_{r}, n_{\theta}\right)},
$$

where we denote the radiative boundary condition by ( $\mathrm{rad}$ ) to indicate the boundary condition for the self-force. This shows that we obtain half of the self-force by using the radiative metric perturbation. The balance formula by [3] and [14] discusses the infinite time averaged loss of the energy and angular momentum, corresponding to $\dot{\mathcal{E}}^{(\mathrm{ret}) a(0,0)}=\dot{\mathcal{E}}^{(\mathrm{rad}) a(0,0)}$, but the present derivation also includes the Carter constant.

This result suggests why the balance formula was derived without a complicated regularization calculation as was required in the self-force calculation. Because the radiative Green function is defined as $\mathbf{G}^{\text {rad }}=\left(\mathbf{G}^{\text {ret }}-\mathbf{G}^{\text {adv }}\right) / 2$ [14], the singular parts of the retarded Green function and the advanced Green function cancel each other; thus, the regularization calculation is naturally done in the balance formula [3] and the radiation reaction formula [13].

Using the general form of the self-force (4.18), one can derive a general property of the orbit with the radiation reaction effect by integrating (4.10), (4.11) and (4.17). Here, it is important to observe that the self-force is derived by the linear metric perturbation. The divergence of the linearized Einstein tensor is known to vanish algebraically; thus, the stressenergy tensor of its source term is conserved in the background. For this reason, the use of a linear metric perturbation as an approximation of the geometry is valid only when the deviation from a geodesic is small enough, and, in [13], we consider the orbit by a perturbation from a geodesic. By this perturbative analysis of the orbit, we find the evolution of the "primary constants' and the 'secondary constants' by the gauge condition (4.16) can be written as

$$
\begin{aligned}
& \mathcal{E}^{a}=\mathcal{E}_{0}^{a}+\left\langle\dot{\mathcal{E}}^{a}\right\rangle \lambda+\sum_{n_{r}, n_{\theta}} \mathcal{E}^{a\left(n_{r}, n_{\theta}\right)} \exp \left[\mathrm{i} n_{r} \chi_{r}+\mathrm{i} n_{\theta} \chi_{\theta}\right], \\
& \lambda^{b}=\lambda_{0}^{b}+\left\langle\ddot{\lambda}^{b}\right\rangle \lambda^{2} / 2+\sum_{n_{r}, n_{\theta}}\left(\dot{\lambda}^{b\left(n_{r}, n_{\theta}\right)} \lambda+\lambda^{b\left(n_{r}, n_{\theta}\right)}\right) \exp \left[\mathrm{i} n_{r} \chi_{r}+\mathrm{i} n_{\theta} \chi_{\theta}\right], \\
& C^{c}=C_{0}^{c}+\left\langle\ddot{C}^{c}\right\rangle \lambda^{2} / 2+\sum_{n_{r}, n_{\theta}}\left(\dot{C}^{c\left(n_{r}, n_{\theta}\right)} \lambda+C^{c\left(n_{r}, n_{\theta}\right)}\right) \exp \left[\mathrm{i} n_{r} \chi_{r}+\mathrm{i} n_{\theta} \chi_{\theta}\right],
\end{aligned}
$$

where $\mathcal{E}_{0}^{a}, \lambda_{0}^{b}, C_{0}^{c}$ are the initial values of the 'constants'. It is notable that $\left\langle\dot{\mathcal{E}}^{a}\right\rangle,\left\langle\ddot{\lambda}^{b}\right\rangle$ and $\left\langle\ddot{C}^{c}\right\rangle$ determine the dominant contributions of (4.21), (4.22) and (4.23), which are derived only by the radiative metric perturbation. We also prove that these quantities are gauge invariant in the physically reasonable class of gauge conditions. 
Because the radiative Green function is a homogeneous Green function, there is a convenient method to calculate it even in a Kerr black hole [9] in the so-called radiation gauge conditions. A necessary calculation here is essentially a minor extension of that used for the balance formula, and we consider that a numerical method is already established [2].

\subsection{Self-force calculation versus radiation reaction formula}

Here we try to compare these two approaches to calculating the orbit. There could be two issues for comparison;

(1) Theoretical issue; whether the resulting orbit is physically acceptable or not

(2) Practical issue; whether the approach is practically available for generating templates for the LISA project.

At this moment, we may say that both the self-force calculation and the radiation reaction formula still face the theoretical issue.

The gravitational radiation reaction is a physically real object. Because the momentum flux carried by gravitational waves is well defined in the asymptotic flat region of the background spacetime, it is reasonable to expect the orbital evolution must be consistent with this effect. There is a widespread belief that the self-force includes the effect of the gravitational radiation reaction. Although this looks a reasonable conclusion, we find that the relation between the gravitational self-force and the gravitational radiation reaction in a usual metric perturbation scheme is not trivial because of the gauge freedom. As we shall argue in section 5, the self-force totally depends on a gauge condition in the whole time interval where a usual metric perturbation scheme is valid; thus the gravitational self-force does not necessarily describe the effect of gravitational radiation reaction to the orbit as we have expected. In section 6 , we shall discuss that this contradicting problem appears because the usual metric perturbation scheme is not appropriate to consider radiation reaction to the orbital evolution, and we shall propose an adiabatic approximation of the metric perturbation where the physically reasonable class of gauge conditions is adopted. Unless the self-force calculation is done in the physically reasonable class of gauge conditions, we consider it necessary to argue that the self-force calculation in a gauge condition describes an orbital evolution by radiation reaction in an appropriate manner.

On the other hand, the radiation reaction formula is derived consistently with the balance formula by using the physically reasonable class of gauge conditions. however, the radiation reaction formula gives us only the infinite time averaged part of the self-force acting on the 'primary constants'. Here we have a question. A general form of the self-force (4.18) shows that the self-force has an oscillating component other than the infinite time averaged component. It is not clear how this oscillating component contributes to the orbital evolution. Equations (4.21), (4.22) and (4.23) show that the infinite time averaged component dominantly determines the orbital evolution; however, this analysis is based on a usual metric perturbation scheme. As we shall argue in section 5, the usual metric perturbation is not appropriate to consider the orbital evolution, and we shall propose an adiabatic approximation of the metric perturbation in section 6 . Then the question remains on the possibility that the oscillating component might be important to consider the orbital evolution.

As we shall discuss in section 6, we consider the radiation reaction formalism under the adiabatic approximation of the metric perturbation. We find that the infinite time averaged part of the self-force actually determines the orbit during the time scale where the adiabatic approximation of the metric perturbation holds. We also find that the oscillating part of the self-force can be eliminated by a special choice of gauge condition in [16]. 
As for the practical issue, the self-force calculation still has a lot to investigate. There are a number of studies of a linear metric perturbation induced by a point particle [2]. However, most of these studies calculate gravitational waves at infinity where both a numerical and a semi-analytic method are established. On the other hand, the self-force calculation needs a mode decomposition of a linear metric perturbation along the orbit in order to derive the bare self-force field (4.5) and a reliable technique for this calculation has not been demonstrated in general.

If the background is a Schwarzschild black hole, one can use the Zerilli-Regge-Wheeler formalism [17] to derive a mode decomposition of the bare self-force field for a general orbit in principle, and we have a result for a circular orbit or a radial orbit, not for a general orbit. But when the background is a Kerr black hole, a method to calculate a metric perturbation has just been proposed [8], and the self-force calculation with this idea is not yet formulated. A more crucial problem here is that it is not clear whether the self-force calculation using this metric perturbation would describe the effect of gravitational radiation reaction in a physically reasonable manner because the gauge condition along the orbit is not well understood.

We also comment that the self-force calculation is a point splitting regularization, and that the singular self-force field might not be optimally subtracted from the bare self-force field. As a result, numerical convergence of the self-force calculation might not be fast in general.

On the other hand, the radiation reaction formula can be seen as a non-trivial extension of the balance formula. We only need a radiative metric perturbation and a convenient method for this calculation is known [9]. Various numerical codes of the balance formula have already been made successfully [2] for either a circular orbit or an equatorial orbit around a Kerr black hole, and we are coming to have a result for a general orbit; therefore, we consider this approach is practically promising. There is also a semi-analytic technique known which may substantially increase the efficiency of the code [18]. We also comment that we have the most optimal convergence in this approach.

\section{Self-force? A myth?}

So far, we frequently noted that the self-force does not necessarily include the effect of the gravitational radiation reaction, which is obviously against our motivation to study the selfforce problem. Because it has not been seriously considered whether the self-force includes the effect of gravitational radiation reaction, we shall devote this section to discussing this problem.

The gravitational radiation reaction is physically real since we can define a momentum flux of gravitational waves in the asymptotic flat region of the background metric; however, we find that the self-force is entirely gauge dependent and that it could vanish by a special gauge choice along the orbit. It is well known that the self-force could vanish at an orbital point by a gauge choice, but we considered this might not be a problem because the selfforce would have non-vanishing components of gravitational radiation reaction by taking an infinite time average of the self-force. In section 4.2, we discuss that this is the case in the physically reasonable class of gauge conditions and that non-vanishing components are proven to be gauge invariant in this gauge class. However, a usual metric perturbation scheme can describe gravitational evolution of the system only for a finite time interval, and, in this whole time interval, the self-force could even vanish by a gauge choice along the orbit as we show in section 5.1. This suggests that the relation between the self-force and the gravitational radiation reaction is entirely gauge dependent, and is not trivial as we expected before. Then we have questions; how is the self-force related to gravitational radiation reaction, and what 
gauge conditions should we take to calculate a self-force? We consider these questions in section 5.2.

\subsection{Metric perturbation}

We suppose that a regularization calculation for a self-force is formally possible by a matched asymptotic expansion as we discuss in section 3.3, and consider only a metric of the far-zone expansion. The metric of the far-zone expansion is a sum of a regular vacuum background metric and its metric perturbation induced by a point particle moving on the background metric. We suppose the point particle has an appropriate structure [7] so that the metric of the far-zone expansion consistently matches a metric of the near-zone expansion.

Suppose we use a usual metric perturbation scheme for the calculation of the metric of the far-zone expansion. We expand the metric and the stress-energy tensor of the point particle with a small parameter $m / L$ as

$$
\begin{aligned}
& g_{\mu \nu}=g_{\mu \nu}^{(b g)}+(m / L) h_{\mu \nu}^{(1)}+(m / L)^{2} h_{\mu \nu}^{(2)}+\cdots, \\
& T^{\mu \nu}=(m / L) T^{(1) \mu \nu}+(m / L)^{2} T^{(2) \mu \nu}+\cdots,
\end{aligned}
$$

where $g_{\mu \nu}^{(b g)}$ is the vacuum background metric. For a valid perturbation, we have

$$
\begin{aligned}
& O(1)>(m / L) h_{\mu \nu}^{(1)}>(m / L)^{2} h_{\mu \nu}^{(2)}>\cdots, \\
& O(1)>(m / L) T^{(1) \mu \nu}>(m / L)^{2} T^{(2) \mu \nu}>\cdots .
\end{aligned}
$$

One can expand the Einstein equation with $m / L$, and we schematically have

$$
\begin{aligned}
& G^{(1) \mu \nu}\left[h^{(1)}\right]=T^{(1) \mu \nu}, \\
& G^{(1) \mu \nu}\left[h^{(2)}\right]+G^{(2) \mu \nu}\left[h^{(1)}, h^{(1)}\right]=T^{(2) \mu \nu}, \\
& \cdots,
\end{aligned}
$$

where $G^{(1) \mu \nu}[h]$ and $G^{(2) \mu v}[h]$ are the terms linear and quadratic in $h_{\mu \nu}$ of the Einstein tensor $G^{\mu v}[g+h]$, respectively.

As we discuss in section 3.3, $T^{(1) \mu v}$ is the usual stress-energy tensor of a monopole particle. It is important to note that the linearized Einstein equation is algebraically divergence free with respect to the background metric as $G^{(1) \mu \nu} ; \nu=T^{(1) \mu \nu}{ }_{j \nu}=0$; thus, in this metric perturbation scheme, the particle as a source of $h_{\mu \nu}^{(1)}$ must move along a geodesic in the background metric $g_{\mu \nu}^{(b g)}$ for a consistent solution of (5.5). An explicit form of $T^{(2) \mu v}$ may be derived by a matched asymptotic expansion of matrices of the far-zone expansion and the near-zone expansion to this order. But, at least one may consider that it has a term of the monopole particle deviating from a geodesic because one can derive the MiSaTaQuWa self-force from the LHS of (5.6) by a mass renormalization [1]. Thus, for the expansion of the stress-energy tensor, we must also consider the expansion of the orbit as

$$
z^{\mu}(\tau)=z^{(b g) \mu}(\tau)+(m / L) z^{(1) \mu}(\tau)+\cdots,
$$

where $\tau$ is defined to be a proper time in the background metric and $z^{(b g) \mu}$ is a geodesic of the background metric. For a valid perturbation, we have

$$
O(1)>(m / L) z^{(1) \mu}>\cdots .
$$


Intuitively speaking, the orbit deviates from a geodesic by the gravitational radiation reaction, and eventually condition (5.8) would be violated, then the metric perturbation in this perturbation scheme would fail to approximate the system.

We consider a gauge transformation on the time scale where the metric perturbation still approximates the system. The gauge transformation is defined by a small coordinate transformation $x^{\mu} \rightarrow \bar{x}^{\mu}=x^{\mu}+(m / L) \xi^{\mu}(x)$ and the orbit transforms as $z^{\mu}(\tau) \rightarrow \bar{z}^{\mu}(\tau)=$ $z^{\mu}\left(\tau^{\prime}\right)+(m / L) \xi^{\mu}\left(z\left(\tau^{\prime}\right)\right)$, where $\tau^{\prime}$ is related to the orbital parameter $\tau$ so that $\tau$ remains a proper time of the new orbit $\bar{z}^{\mu}(\tau)$ in the background metric. Applying this to the perturbation expansion of the orbit (5.7), we find the gauge transformation of $(m / L) z^{(1) \mu}$ as

$(m / L) z^{(1) \mu}(\tau) \rightarrow(m / L) \bar{z}^{(1) \mu}(\tau)=(m / L) z^{(1) \mu}(\tau)+v^{(b g) \mu}\left(\tau^{\prime}-\tau\right)+(m / L) \xi^{\mu}\left(z^{(b g)}(\tau)\right)$,

where $v^{(b g) \mu}=\mathrm{d} z^{(b g) \mu} / \mathrm{d} \tau$. We find that we could eliminate $(m / L) z^{(1) \mu}(\tau)$ if the gauge transformation along the orbit satisfies

$$
(m / L) \xi^{\mu}\left(z^{(b g)}(\tau)\right)=-(m / L) z^{(1) \mu}(\tau)+v^{\mu} \delta \tau,
$$

with an arbitrary small function $\delta \tau \sim O(m / L)$. There exists a gauge transformation which satisfies this condition for the whole time interval where the metric perturbation scheme is valid because it is smaller than $O(1)$, and the orbit becomes a geodesic of the background metric in this gauge condition.

Because the self-force is a self-acceleration to deviate the orbit from a geodesic, $(m / L) z^{(1) \mu}(\tau)=0$ in this gauge condition means that the self-force entirely vanishes for the whole time interval where the metric perturbation scheme is valid. This extreme example suggests that the self-force is a totally gauge-dependent object in the usual metric perturbation scheme, and even a time average of the self-force over the longest time scale where the metric perturbation is valid is gauge dependent in general.

The essential reason why the self-force could not include the effect of gravitational radiation reaction is that this perturbation scheme only allows a small deviation from a geodesic as in (5.8). Because of this, one could always eliminate the deviation by a gauge transformation as in (5.9). We note that this problem may not be solved by calculating a nonlinear metric perturbation because the problem comes from the metric perturbation scheme. We consider a solution of this problem is to modify the metric perturbation scheme so that one can describe a non-perturbative orbital deviation from a geodesic. If the orbit can deviate from a geodesic non-perturbatively, one cannot eliminate it by a gauge transformation.

\subsection{Energetics of the orbit and radiation}

A hint to understand why such an unexpected thing happens can be seen in [13]. In section 3 of [13], we argue that the orbital energy does not decrease monotonically by the emission of gravitational waves. We consider that gravitational radiation has its own energy and that the self-force describes the interaction between the orbital energy and the radiation energy. In fact, one can define an effective stress-energy tensor of the orbit and gravitational radiation[1]. As in the previous subsection, we only consider the metric of the far-zone expansion, and we suppose that the metric is a sum of the vacuum background metric and its perturbation induced by a point particle with an appropriate internal structure [7] as $g_{\mu \nu}=g_{\mu \nu}^{(b g)}+h_{\mu \nu}$.

The Einstein equation can formally be written as $G_{\mu \nu}^{(1)}[h]+G_{\mu \nu}^{(2+)}[h]=T_{\mu \nu}$, where $G_{\mu \nu}^{(1)}$ and $G_{\mu \nu}^{(2+)}$ are the linear terms and the rest of the Einstein tensor with respect to $h_{\mu \nu}$, and $T_{\mu \nu}$ is the stress-energy tensor of the point particle. Because $G_{\mu \nu}^{(1)}$ is algebraically divergence free, one can define a conserved stress-energy tensor in the background metric by

$$
\mathcal{T}^{\mu \nu}=T^{\mu \nu}-G^{(2+) \mu \nu}[h] .
$$


We consider that the first term and the second term of (5.11) represent the stress-energy tensors of the particle and gravitational radiation respectively, and each of these are not conserved in the background by themselves. We suppose that the background metric $g_{\mu \nu}^{(b g)}$ is a Kerr black hole and has a timelike Killing vector $\xi_{\mu}$, then one can define a total orbital and radiation energy in the background metric as

$$
E^{(\mathrm{tot})}=E^{(\mathrm{orb})}+E^{(\mathrm{rad})}, \quad E^{(\mathrm{orb})}=-\int \mathrm{d} \Sigma_{\mu} \xi_{\nu} T^{\mu \nu}, \quad E^{(\mathrm{rad})}=\int \mathrm{d} \Sigma_{\mu} \xi_{\nu} G^{(2+) \mu \nu},
$$

where the surface integration is taken over a spacelike hypersurface bounded by the future horizon and the future null infinity of the background black-hole metric. We note that the radiation energy $E^{(\mathrm{rad})}$ is not necessarily positive.

By integrating (5.11) over a small world tube surface around the orbit, one can derive the MiSaTaQuWa self-force [1], which shows that the self-force describes the interaction between the orbital energy and the gravitational radiation energy. A gauge condition for the self-force could be interpreted as a small arbitrariness in defining the orbital energy and the radiation energy in (5.12) [16]. In order to see this, we apply the metric perturbation scheme of section 5.1 to (5.12), and we have

$$
\begin{aligned}
& E^{(\mathrm{orb})}=(m / L) E^{(\mathrm{orb})(1)}+(m / L)^{2} E^{(\mathrm{orb})(2)}+\cdots, \\
& (m / L) E^{(\mathrm{orb})(1)}=-\int \mathrm{d} \Sigma_{\mu} \xi_{v}(m / L) T^{(1) \mu \nu}, \\
& (m / L)^{2} E^{(\mathrm{orb})(2)}=-\int \mathrm{d} \Sigma_{\mu} \xi_{v}(m / L)^{2} T^{(2) \mu \nu}, \cdots \\
& E^{(\mathrm{rad})}=(m / L)^{2} E^{(\mathrm{orb})(2)}+\cdots, \\
& (m / L)^{2} E^{(\mathrm{rad})(2)}=\int \mathrm{d} \Sigma_{\mu} \xi_{v}(m / L)^{2} G^{(2) \mu v}\left[h^{(1)}, h^{(1)}\right], \ldots
\end{aligned}
$$

In the usual perturbation scheme, $(m / L) T^{(1) \mu \nu}$ conserves by itself; as a result, $E^{(\mathrm{orb})(1)}$ becomes a constant of motion. By a gauge transformation around the orbit, one can show that the orbital energy and the radiation energy transform as

$$
\begin{aligned}
& (m / L)^{2} E^{(\mathrm{orb})(2)} \rightarrow(m / L)^{2} \bar{E}^{(\mathrm{orb})(2)}=(m / L)^{2} E^{(\mathrm{orb})(2)}+(m / L)^{2} \delta E, \\
& (m / L)^{2} E^{(\mathrm{rad})(2)} \rightarrow(m / L)^{2} \bar{E}^{(\mathrm{rad})(2)}=(m / L)^{2} E^{(\mathrm{rad})(2)}-(m / L)^{2} \delta E,
\end{aligned}
$$

where $\delta E$ is arbitrary, and the orbital energy is entirely gauge dependent by itself as we discuss in the previous section.

Although we do not yet propose a new metric perturbation scheme, we may extend the argument of the orbital energy and the radiation energy since they are defined in a nonperturbative manner as (5.12). The balance formula is derived by gravitational waves at the future null infinity and the future horizon of the background black-hole geometry, and it describes radiation reaction to the total energy rather than the orbital energy [16]. In the usual metric perturbation scheme, one can decrease only the radiation energy by radiation reaction while keeping the orbital energy constant by the gauge freedom. Although this is mathematically allowed in the usual metric perturbation scheme, if the system continues to lose total energy by radiation reaction, it will eventually be difficult to understand the orbit in a physically reasonable way. Since we keep the orbital energy constant, the radiation energy will inevitably decrease substantially by radiation reaction; as a result, the amplitude of the metric perturbation would be non-perturbatively large and one cannot use the coordinate system of the background metric as a reference to observe the orbit. 
In this case, only numerical relativity may be able to describe the evolution of the system, and, even if it is possible, it may be difficult to understand the system. Thus, for this technical advantage, we consider it reasonable to keep the amplitude of the metric perturbation small and we should decrease the orbital energy by radiation reaction rather than the radiation energy. We consider that this would be a reasonable least criterion for a convenient gauge condition to calculate the self-force. Although we do not have a simpler criterion for a convenient gauge condition, the physically reasonable class of gauge conditions (4.16) satisfies this criterion. Because the self-force in this gauge class is consistent with the balance formula, only the orbital energy decreases by radiation reaction and the radiation energy is just oscillating, as a result, the metric perturbation would not grow non-perturbatively large.

Although the argument of energetics shows us the importance of the self-force, this is just a qualitative argument. In the next section, we propose a new perturbation scheme and we formulate a method to calculate the gravitational evolution of an extreme mass ratio binary in this picture.

\section{Adiabatic extension}

In this section, we review our recent progress on the radiation reaction formalism in [16]. We discuss in section 5 that the self-force does not necessarily include the effect of the gravitational radiation reaction because of the perturbation scheme. In order to break this limitation, we introduce an adiabatic approximation to this problem. The adiabatic approximation is well known in classical mechanics; however, the application to gauge field theory such as a gravitational perturbation is not so common.

\subsection{Adiabatic metric perturbation}

From the result of the radiation reaction formula, the orbital deviation becomes $O(1)$ when $\lambda \sim O\left((m / L)^{-1 / 2}\right)$. This time scale is called the dephasing time of a orbit. The usual metric perturbation scheme is valid only within this time scale as we discuss in section 5 . Our purpose here is to extend this time scale of validity by modifying the metric perturbation scheme. For an explicit discussion, we use the physically reasonable class of gauge conditions (4.16).

We denote the orbital constants of a geodesic by $\gamma=\left\{\mathcal{E}^{a}, \lambda^{b}, C^{c}\right\}$. Because a linear metric perturbation is induced by a geodesic, it is a function of $\gamma$ as $h_{\mu \nu}(x)=h_{\mu \nu}(\gamma ; x)$. In the radiation reaction formula [13], we use an adiabatic approximation to the orbit. In this approximation, we approximate the orbit by a geodesic at each instant, and consider the evolution of the 'orbital constants' by the effect of gravitational radiation reaction; thus, we may write the 'orbital constants' as functions of the orbital parameter as $\gamma(\lambda)$. We consider extending this idea to the metric perturbation. We foliate the spacetime by spacelike hypersurfaces which intersect with the orbit. We define the foliation function $f(x)$ by the orbital parameter at the intersection of the orbit and the surfaces as $f(z(\lambda))=\lambda$. We define an adiabatic linear metric perturbation by using the linear metric perturbation on the foliation surface induced by the geodesic $\gamma(f)$ as

$$
h_{\mu \nu}^{a d}(x)=h_{\mu \nu}(\gamma(f) ; x)
$$

Here we do not specify an explicit form of the foliation function; as a result, an adiabatic linear metric perturbation is not defined uniquely. We consider that we may need a constraint of the foliation function for an adiabatic nonlinear metric perturbation; however, it does not change the result of the following discussion. We consider that the adiabatic linear metric perturbation is a non-trivial extension of the linear metric perturbation. 
Under the physically reasonable class of gauge conditions, a formal expression of the tensor Green function becomes

$G_{\mu \nu \mu^{\prime} \nu^{\prime}}\left(x, x^{\prime}\right)=\sum_{\omega, m} g_{\mu \nu \mu^{\prime} \nu^{\prime}}^{(\omega, m)}\left(r, \theta ; r^{\prime}, \theta^{\prime}\right) \exp \left[-\mathrm{i} \omega\left(t-t^{\prime}\right)+\mathrm{i} m\left(\phi-\phi^{\prime}\right)\right]$.

This gives a general form of the linear metric perturbation in this class of gauge conditions induced by a geodesic $\gamma$ as

$h_{\mu \nu}(\gamma ; x)=\sum_{\omega, m, n_{r}, n_{\theta}} k^{\left(\omega, m, n_{r}, n_{\theta}\right)}(\gamma) h_{\mu \nu}^{\left(\omega, m, n_{r}, n_{\theta}\right)}\left(\mathcal{E}^{a} ; r, \theta\right) \exp [-\mathrm{i} \omega t+\mathrm{i} m \phi]$,

$k^{\left(\omega, m, n_{r}, n_{\theta}\right)}(\gamma)=\int \mathrm{d} \lambda \tilde{k}^{\left(\omega, m, n_{r}, n_{\theta}\right)}\left(\mathcal{E}^{a}\right) \exp \left[\mathrm{i} \omega \kappa_{t}-\mathrm{i} m \kappa_{\phi}-\mathrm{i} n_{r} \chi_{r}-\mathrm{i} n_{\theta} \chi_{\theta}\right]$.

We construct the adiabatic linear metric perturbation (6.1) with the general form of the linear metric perturbation (6.3). When we operate the linearized Einstein operator, we have

$$
G_{\mu \nu}^{(1)}\left[h^{a d}\right]=T_{\mu \nu}[\gamma(f)]+\Lambda_{\mu \nu}^{(1)}\left[h^{a d}\right]
$$

where an extra term $\Lambda_{\mu \nu}^{(1)}$ appears because the adiabatic linear metric perturbation is not induced by a geodesic of the background. It is notable that $T^{\mu \nu}[\gamma(f)]$ is a stress-energy tensor of a point particle moving along the orbit with the effect of gravitational radiation reaction.

The adiabatic metric linear perturbation solves the Einstein equation to an accuracy of $O(m / L)$ as long as $O(m / L)>\Lambda_{\mu \nu}^{(1)}$ holds. Since $\Lambda_{\mu \nu}^{(1)}$ is proportional to the $\lambda$-derivatives of the orbital 'constants' $\gamma(\lambda)$, the validity of the adiabatic metric perturbation depends on how the orbit evolves by a self-force. In the next section, we discuss the orbital evolution under the physically reasonable class of gauge conditions. We find that the adiabatic metric perturbation is a well-approximated solution of the linearized Einstein equation in the radiation reaction time scale $O\left((m / L)^{-1}\right)>\lambda$; hence, the time scale of validity is much longer than that of the usual metric perturbation scheme.

\subsection{Adiabatic evolution of the orbit}

Different from the usual perturbation scheme one cannot use the perturbation of the orbit any more, and we need to calculate the orbit in a non-perturbative manner. Because of a technicality of a non-perturbative calculation, we only summarize the result and suggest the readers refer to [16] for the details.

The self-force defined by the adiabatic linear metric perturbation is simply derived as

$$
\frac{\mathrm{d}}{\mathrm{d} \lambda} \mathcal{E}^{a}=\tilde{F}^{a}\left(\mathcal{E}^{a}(\lambda), \lambda^{b}(\lambda), C^{c}(\lambda) ; \lambda\right)+O\left((m / L)^{2}\right),
$$

and we can use the result of the original radiation reaction formula [13] in the radiation reaction time scale $\lambda<O\left((m / L)^{-1}\right)$. As for the 'primary constants', one can still deal with the evolution by a perturbation, and we find that $\dot{\mathcal{E}}^{a(0,0)}$ dominantly determines the orbital evolution. The subdominant parts of the 'primary constants' are $O(m / L)$ and we may not see these effects by gravitational waves. The evolution of the 'secondary constants' becomes non-perturbative beyond the dephasing time; however, we find the perturbation results of (4.22) and (4.23) are qualitatively correct. The dominant part of their evolution is described only by $\mathcal{E}^{a(0,0)}$, and the subdominant part grows linearly in $\lambda$. 
In summary, we find the qualitative behaviour of the orbital evolution as

$\frac{\mathrm{d}}{\mathrm{d} \lambda} \mathcal{E}^{a}=O(m / L), \quad \frac{\mathrm{d}}{\mathrm{d} \lambda} \lambda^{b}=O((m / L) \lambda), \quad \frac{\mathrm{d}}{\mathrm{d} \lambda} C^{c}=O((m / L) \lambda)$.

This gives us the qualitative estimate of (6.5) and we find $\Lambda_{\mu \nu}^{(1)}=O\left((m / L)^{2} \lambda\right)$. Hence, the adiabatic linear metric perturbation is valid in the radiation reaction time scale $O\left((m / L)^{-1}\right)>\lambda$.

\subsection{Radiation reaction gauge}

The validity of the adiabatic linear metric perturbation depends on the behaviour of $\Lambda_{\mu \nu}^{(1)}$ in (6.5). Because $\Lambda_{\mu \nu}^{(1)}$ is gauge dependent, we may consider extending the validity by using a remaining gauge freedom in the physically reasonable class of gauge conditions.

Using the adiabatic linear metric perturbation, we find that the 'primary constants' still evolve perturbatively as

$$
\mathcal{E}^{a}=\mathcal{E}_{0}^{a}+\left\langle\dot{\mathcal{E}}^{a}\right\rangle \lambda+\sum_{n_{r}, n_{\theta}} \mathcal{E}^{a\left(n_{r}, n_{\theta}\right)} \exp \left[\mathrm{i} n_{r} \chi_{r}+\mathrm{i} n_{\theta} \chi_{\theta}\right]
$$

By a gauge transformation $x^{\mu} \rightarrow x^{\mu}+\xi^{\mu}$, the 'primary constants' transform as $\mathcal{E}^{a} \rightarrow \mathcal{E}^{a}+\delta \mathcal{E}^{a}$ where

$$
\begin{aligned}
& \delta \mathcal{E}^{E / L}=-\eta_{\alpha}^{E / L} v^{\beta} \xi_{; \beta}^{\alpha}+\eta_{\alpha ; \beta}^{E / L} v^{\beta} \xi^{\alpha}, \\
& \delta \mathcal{E}^{C}=-\eta_{\alpha \beta}^{C} v^{\beta} v^{\gamma} \xi_{; \gamma}^{\alpha} .
\end{aligned}
$$

In [16], we find that, by an appropriate gauge choice, one can eliminate the oscillating part of (6.8) as

$$
\mathcal{E}^{a}=\left(\mathcal{E}_{0}^{a}+\delta \mathcal{E}_{0}^{a}\right)+\left\langle\dot{\mathcal{E}}^{a}\right\rangle \lambda+O\left(\mu^{2} t\right) .
$$

Here, one has to introduce a small shift of the initial values $\delta \mathcal{E}_{0}^{a}$ so that the gauge transformation behaves as $\xi=O(m / L)$. In this gauge condition, the self-force has only the radiative part as

$$
\tilde{F}^{a}\left(\mathcal{E}^{a}(\lambda), \lambda^{b}(\lambda), C^{c}(\lambda) ; \lambda\right)=\left\langle\dot{\mathcal{E}}^{a}\right\rangle\left(\mathcal{E}^{a}(\lambda)\right) .
$$

For this reason, we call this by a radiation reaction gauge.

The self-force is expected to have a conservative part as well as a radiative part; however, in this gauge condition, the conservative part is integrated out to be a renormalization of the initial values $\mathcal{E}_{0}^{a} \rightarrow \mathcal{E}_{0}^{a}+\delta \mathcal{E}_{0}^{a}$. We also note that a radiation reaction gauge condition is applicable to an orbit of a spinning particle. It is known that the orbit of the spinning test particle deviates from a geodesic [19] by a coupling of its spin and the curvature of the background metric. This effect can also be renormalized to the initial values in the radiation reaction gauge condition.

Using a post-Newtonian estimation [20], the self-force can be estimated to be $O(m / L \times$ $v^{5}$ ), where $v^{2} \sim 0.1$ around a last stable orbit. This is smaller than that in a general gauge condition of the physically reasonable class. As a result, one can predict the orbit in the time scale $\lambda<O\left(\left(m / L v^{5}\right)^{-1}\right)$, which may correspond to several years for a promising target of LISA.

\section{Summary and future prospect}

It is widely believed that a self-force could describe an orbital evolution with the effect of gravitational radiation reaction. For this reason, the so-called Capra community have been 
trying to develop a method to explicitly calculate the self-force acting on a particle orbiting around a Kerr black hole, and we are having success in developing a regularization calculation method either by the self-force calculation [10] or by the radiation reaction formula [13]. On the other hand, we come to have concern as to what physical information the self-force actually carries because the self-force is gauge dependent. For this concern, it was discussed that it might be necessary to develop a nonlinear metric perturbation to grasp the physical meaning of the self-force.

A second-order metric perturbation may be derived consistently only when we consider an orbit with the effect of gravitational radiation reaction, and it gives us gauge-invariant information by an asymptotic gravitational waveform, for example, by a modulation of a gravitational wave phase. We consider that we may see the radiation reaction effect from the waveform; however, it has nothing to do with the radiation reaction effect on the orbit because radiation reaction to the orbit is purely gauge dependent. We find that one can define a radiation energy, an orbital energy and a total energy as a sum of the radiation and orbital energies. The radiation reaction we usually consider (say, by the balance formula) is a reaction acting on the total energy, but the orbital motion is related to the orbital energy.

In section 5, we discuss that the gauge freedom is interpreted as a small ambiguity in separating the total energy into the radiation energy and the orbital energy. This separation is entirely arbitrary in the usual metric perturbation scheme; however, we discuss that, without some constraint on this separation, we may have difficulty in interpreting the orbit. When we continue the evolution beyond the usual metric perturbation scheme, the metric perturbation would grow non-perturbatively large and, as a result, the background metric cannot be used as an approximated reference to track the orbit. We argue that the physically reasonable class of gauge conditions is, at least, a reasonable gauge choice to avoid such a non-perturbative situation in section 5 , and we show that an adiabatic extension of the linear metric perturbation is possible in section 6 .

A possible question here is whether there is a wider class of gauge conditions which allows an adiabatic extension of a linear metric perturbation. Especially, we introduce the physically reasonable class of gauge conditions because a linear metric perturbation is derived by a Fourier-harmonic decomposition, and it is not clear whether an adiabatic extension of a linear metric perturbation by a time domain calculation is possible or not. If it is not possible, the self-force calculation by the time domain calculation may not predict an orbital evolution in the radiation reaction time scale.

Different from the self-force calculation, the radiation reaction formula conveniently calculates only an infinite time averaged part of the self-force. We find that this part is actually enough for the prediction of an orbit in the radiation reaction time scale. We find the rest of the self-force only makes a small change to the orbit, which can be totally eliminated by a choice of the gauge.

A crucial problem for calculating gravitational waveforms for the LISA project is whether the radiation reaction time scale is sufficiently longer than the observation time of an astrophysically expected target. If we calculate the self-force in the physically reasonable class of gauge conditions, the radiation reaction time scale would be around several months in general. Since an observation time of LISA would be several years, there could be the case that one cannot calculate reliable waveforms. We find that one could modify the radiation reaction time by using the remaining gauge freedom in this class. We propose the so-called radiation reaction gauge where the self-force has only the radiation reaction component, which is derived by the radiation reaction formula. In this gauge condition, we find that we would have reliable waveforms of several years, which is sufficient for the present LISA project. 
Although we consider the radiation reaction formula with an adiabatic linear metric perturbation may be sufficient for the LISA project, it remains a great theoretical challenge to calculate an orbit longer than the radiation reaction time scale.

\section{Acknowledgments}

YM thanks Professor Kip Thorne and Professor Sterl Phinney for encouragement. YM was supported by NASA grant NAG5-12834 and NASA-ATP grant NNG04GK98G at CalTech.

\section{References}

[1] Poisson E 2004 Living Rev. Rel. 76 http://www.livingreviews.org/lrr-2004-6

Detweiler S and Whiting B F 2003 Phys. Rev. D 67024025

Mino Y, Sasaki M and Tanaka T 1997 Prog. Theor. Phys. (Suppl.) 128373

Mino Y, Sasaki M and Tanaka T 1997 Phys. Rev. D 553457

Quinn T C and Wald R M 1997 Phys. Rev. D 563381

[2] Some pioneering works of a perturbation induced by a point particle by numerical methods are summarized in Breuer R A 1975 Gravitational Perturbation Theory and Synchrotoron Radiation, (Lecture Notes in Physics vol 44) (Berlin: Springer) p 1

Chandrasekhar S 1983 Mathematical Theory of Black Holes (Oxford: Oxford University Press)

Nakamura T, Oohara K and Kojima Y 1987 Prog. Theor. Phys. Suppl. 901

Analytic methods are summarized in: Mino Y, Sasaki M, Shibata M, Tagoshi H and Tanaka T 1997 Prog. Theor. Phys. Suppl. 1281

Sasaki M and Tagoshi H 2003 Living Rev. Rel. 66 http://www.livingreviews.org/lrr-2003-6

Recent numerical calculations of high precision are: Glampedakis K and Kennefick D 2002 Phys. Rev. D 66 044002

Hughes S A 2001 Phys. Rev. D 64064004

Hughes S A 2000 Phys. Rev. D 61084004

Hughes S A 2001 Phys. Rev. D 63049902 (erratum)

[3] Teukolsky S A 1973 Astrophys. J. 185635

Press W H and Teukolsky S A 1973 Astrophys. J. 185649

Teukolsky S A and Press W H 1974 Astrophys. J. 193443

[4] Kennefick D and Ori A 1996 Phys. Rev. D 534319

Mino Y, Sasaki M, Shibata M, Tagoshi H and Tanaka T 1997 Prog. Theor. Phys. Suppl. 1281 section 10

[5] DeWitt B S and Brehme R W 1960 Ann. Phys. 9220

[6] D’Eath P D 1975 Phys. Rev. D 111387

Thorne K S and Hartle J B 1985 Phys. Rev. D 311815

[7] Dixon W G 1979 Isolated Gravitational Systems in General Relativity ed J Ehlers (Amsterdam: North-Holland) p 156

[8] Ori A 2003 Phys. Rev. D 67124010

[9] Chrzanowski P L 1975 Phys. Rev. D 112042

[10] Detweiler S, Messaritaki E and Whiting B F 2003 Phys. Rev. D 67104016

Barack L and Ori A 2003 Phys. Rev. Lett. 90111101

Barack L and Ori A 2003 Phys. Rev. D 67024029

Mino Y, Nakano H and Sasaki M 2003 Prog. Theor. Phys. 1081039

Barack L and Lousto C O 2002 Phys. Rev. D 66061502

Barack L and Ori A 2002 Phys. Rev. D 66084022

Barack L, Mino Y, Nakano H, Ori A and Sasaki M 2002 Phys. Rev. Lett. 88091101

[11] Mino Y and Nakano H 1998 Prog. Theor. Phys. 100507

[12] Barack L 2000 Phys. Rev. D 62084027

Barack L and Burko L M 2000 Phys. Rev. D 62084040

Barack L and Ori A 2000 Phys. Rev. D 61061502

Burko L M 2000 Phys. Rev. Lett. 844529

[13] Mino Y 2003 Phys. Rev. D 67084027

[14] Gal'tsov D V 1982 J. Phys. A: Math. Gen. 153737

[15] Chandrasekhar S 1983 The Mathematical Theory of Black Holes (Oxford: Oxford University Press) 
[16] Mino Y Prog. Theor. Phys. submitted

[17] Zerilli F J 1970 Phys. Rev. D 22141

[18] Fujita R and Tagoshi H 2004 Prog. Theor. Phys. 112415

Mano S and Takasugi E 1997 Prog. Theor. Phys. 97213

Mano S, Suzuki H and Takasugi E 1996 Prog. Theor. Phys. 951079

[19] Papapetrou A 1951 Proc. R. Soc. Lond. A 209243

[20] Blanchet L 2002 Living Rev. Rel. 53 and the reference therein 